\title{
Dejerine-Sottas disease with sensorineural hearing loss, nystagmus, and peripheral facial nerve weakness: de novo dominant point mutation of the PMP22 gene
}

\author{
V V Ionasescu, C Searby, S A Greenberg
}

\begin{abstract}
A 32 year old woman with Dejerine-Sottas disease and negative family history is reported. Clinical onset of her condition was with congenital weakness of her distal four extremities, accompanied by peripheral facial nerve weakness, deafness, and nystagmus. She has used a wheelchair all her life. Sural nerve biopsy showed proliferation of Schwann cells, extensive endoneural fibrosis, axon loss, and demyelination. MNCVs showed marked slowing. MRI of the brain was normal. Molecular genetic studies indicated a de novo dominant missense point mutation of exon 3 of the peripheral myelin protein 22 gene at nucleotide 264 causing replacement of serine with leucine.

( $f$ Med Genet 1996;33:1048-1049)
\end{abstract}

Key words: Dejerine-Sottas disease; point mutation; PMP22 gene.

This article reports a case of Dejerine-Sottas disease (DSD) that corresponds to the basic clinical and electrophysiological criteria ${ }^{1-3}$ who had, in addition, nystagmus, bilateral sensorineural hearing loss, and bilateral peripheral facial nerve weakness. Molecular genetic analysis showed a de novo dominant mutation of the peripheral myelin 22 (PMP22) gene.

\section{Case report}

A 32 year old woman with DSD was born in the Philippines and has lived in San Francisco since the age of 15 . The family history (both parents and sibs) is negative for DSD and hearing loss. She was significantly developmentally delayed with congenital weakness of her distal four extremities accompanied by facial nerve weakness, hearing loss, and nystagmus. She walked with help at the age of 3 and has used a wheelchair all her life. She has remained relatively stable for the first two decades, but has noticed more weakness of her hands over the last five to 10 years. Medical records from the Shriner's Hospital for Crippled Children and Children's Hospital of San Francisco include a report of a nerve and muscle biopsy done in March 1979 at the age of 15 . The sural nerve was markedly abnormal with many axons showing proliferation of Schwann cells causing a typical onion bulb appearance. In addition, extensive endoneural fibrosis, axon loss, and demyelination consistent with DSD were also reported. The muscle biopsy was normal. Motor nerve conduction velocities (MNCVs) at the time showed marked slowing of ulnar motor conduction with a forearm velocity of $11 \mathrm{~m} / \mathrm{sec}$. The amplitude was not recorded. Nerve conduction studies were repeated at the age of 32 . No motor or sensory potentials (median, ulnar) were obtained, a finding consistent with severe degeneration of motor and sensory nerves. MRI of the brain done on 1.5.90 was normal. Neurological evaluation showed wheelchair confinement with severe weakness of the distal muscles of her extremities and mild weakness of her proximal limb muscles. The patient was areflexic. There was profound loss of vibration and joint position sense in her hands and feet. Limb ataxia was also present and appeared to be sensory in origin. She was unsteady sitting in her wheelchair with her eyes closed. Evaluation of cranial nerves showed coarse horizontal nystagmus at rest and with vertical and lateral eye movements. There was moderate bilateral sensorineural hearing loss and bilateral peripheral facial nerve paresis of lower motor neurone type involving the forehead, the eyelids, and the lips. In addition, the patient had advanced thoracolumbar scoliosis. She has undergone back surgery in the past with placement of Harrington rods.

Molecular genetic studies showed that the patient did not have the duplication associated with Charcot-Marie-Tooth disease type 1A (CMT1A). The absence of the CMT1A duplication was confirmed by pulsed field gel electrophoresis, which did not detect the typical $500 \mathrm{~kb}$ SacII junction fragment. ${ }^{4}$

\section{DETECTION OF THE PMP22 MUTATION}

The amplification of the four coding exons of the PMP22 gene was done by polymerase chain reaction (PCR) from genomic DNA using specific primer sequences and the corresponding PCR products were sequenced directly as previously described. ${ }^{5} \mathrm{~A} \mathrm{C}$ to $\mathrm{T}$ missense point mutation present in the heterozygous state was identified at nucleotide 264 of the cDNA sequence within exon 3 . This point mutation resulted in a change of a serine codon to a leucine codon at amino acid 72 (fig 1). No other mutations were found in PMP22 exons 1,2 , or 4 . 

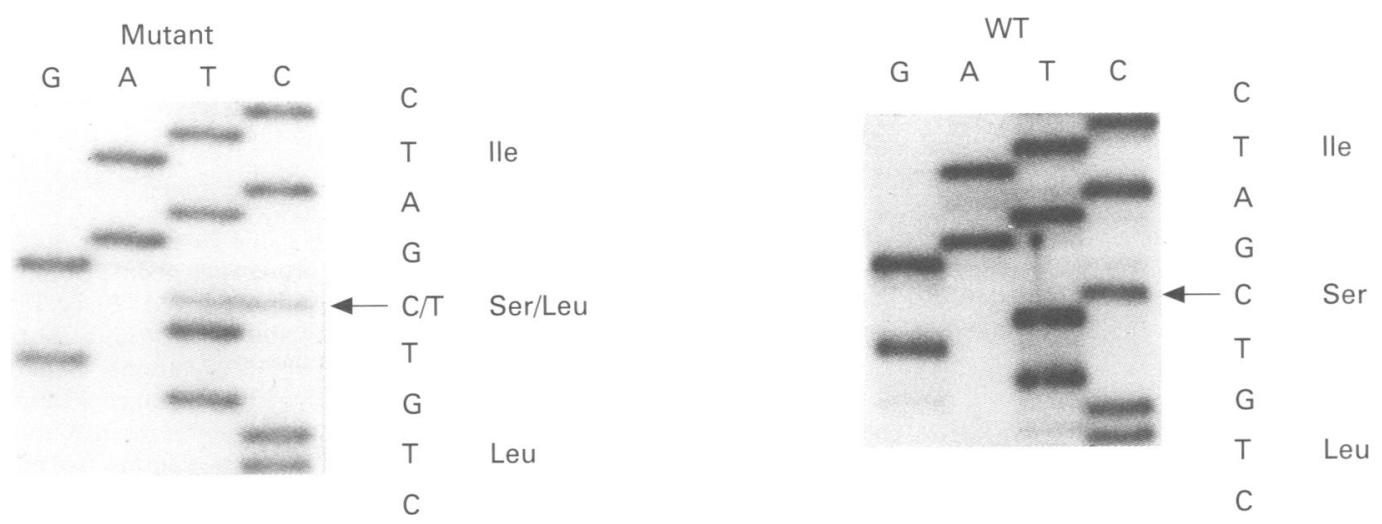

Figure 1 Direct nucleotide sequence determination of biotinylated PCR products corresponding to PMP22 exon 3 was performed. The sense sequences are shown for the proband and an unaffected control. The location of the $C$ to $T$ point mutation is indicated with an arrow. The $C$ to $T$ transition results in the substitution of serine for leucine in the $72 n d$ codon as indicated to the right of the mutant sequence.

\section{Discussion}

Peripheral facial nerve weakness, deafness, and nystagmus developed early in the same period of time as the distal weakness of her four extremities, suggesting the same aetiology. This evidently does not rule out the possibility of acquired deafness, nystagmus, and peripheral facial nerve weakness. However, the early bilateral involvement of VII and VIII cranial nerves supports more a degenerative genetic process than acquired pathology.

Dyck et $a l^{1}$ defined DSD as a severe infantile demyelinating motor and sensory neuropathy with very low MNCVs. Sensory hearing loss was mentioned in only one case. ${ }^{1}$ Nystagmus and peripheral facial nerve weakness have not been reported in this condition. The original description of the disease in a pair of affected sibs $^{2}$ suggested autosomal recessive inheritance. However, molecular genetic studies done in five cases has shown a heterogeneous disease with autosomal dominant mutations of the PMP22 gene as reported by Roa et at in two cases and by Ionasescu et $a l^{t}$ in one case and of the myelin Po gene as reported by Hayasaka $e t$ al in two unrelated cases.

The interest of our new case is the presence of clinically documented cranial nerve involvement with the same point mutation of the PMP22 gene as previously reported. ${ }^{56}$

1 Dyck PJ, Chance P, Lebo R, Carney AJ. In: Dyck PJ, et al, eds. Peripheral neuropathy. Philadelphia: Saunders, 1993: 1094-136.

2 Dejerine J, Sottas J. Sur la nevrite interstitielle, hypertrophique et progressive de l'enfance. $C R$ Soc Biol Paris 1893;45:63-96.

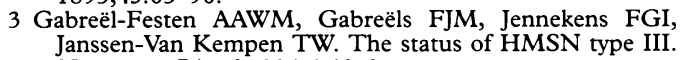
Neuromusc Disord 1994;4:63-9.

4 Lupski JR, Montes de Oca-Luna, Slaugenhaupt S, et al. DNA duplication associated with Charcot-Marie-Tooth disease type 1A. Cell 1991;66:219-32.

5 Roa BB, Dyck PJ, Marks HG, Chance PF, Lupski JR. Dejerine-Sottas syndrome associated with point mutation in jerine-Sottas syndrome associated with point mutation in the peripheral m.
$1993 ; 5: 269-73$.

6 Ionasescu V, Ionasescu R, Searby C, Neahring R. DejerineSottas disease with de novo dominant point mutation of the PMP22 gene. Neurology 1995;45:1766-7.

7 Hayasaka K, Himoro M, Sawaishi Y, et al. De novo mutation of the myelin Po gene in Dejerine-Sottas disease (hereditary motor and sensory neuropathy type III). Nat Genet 1993; 5:266-8. 